\title{
The Compatibility of Downward Causation and Emergence
}

\author{
Simone Gozzano \\ Università dell'Aquila - Italy
}

\begin{abstract}
In this paper, I shall argue that both emergence and downward causation, which are strongly interconnected, presuppose the presence of levels of reality. However, emergence and downward causation pull in opposite directions with respect to my best reconstruction of what levels are. The upshot is that emergence stresses the autonomy among levels while downward causation puts the distinction between levels at risk of a reductio ad absurdum, with the further consequence of blurring the very notion of downward. Therefore, emergence and downward causation are not fit to each other vis-a-vis the concept of level.
\end{abstract}

Keywords: emergent properties; law; level; downward causation; causal power.

\section{First steps into the issue}

It seems somehow intuitive to posit different levels of reality. We see entities having different properties, being these physical, chemical or biological, and so we think of these properties as belonging to different levels or layers. These levels turn out as corresponding to the topics of different scientific disciplines, such as physics, chemistry, molecular biology, and so up, into something like a ladder or a layer pie -- an image that is reminiscent of XIX century Positivism and which we may refer to as the layered view of reality. The various levels, though, are not thought to be completely independent from one another. After all, many entities have biological properties along with physical or chemical properties. Or, imagining a tighter relation, entities have biological properties in virtue of having chemical or physical properties. So, a relation between levels is needed. Here comes emergence: the sprouting of levels has been construed, by many, in terms of it. For instance, chemical properties emerge from physical properties and biological properties from the chemical or physical ones.

\section{The main aspects of the issue.}

One motivation behind the general intuition of the layered view, is to find a place for mental properties in the physical order. Given the emergence relation, mental properties are thought to be emerging from underlying physical properties, in present terms from neurophysiological properties. So, a property of the mental kind emerges from a property of the physical (neural) kind in that the former depends on the latter but cannot be reduced to or identified with it. The supposed advantage of the emergence relation is twofold: from one side, it guarantees autonomy; on the other side it preserves dependence.

Marc Bedau has stressed that:

1) Emergent properties are somehow constituted by, and generated from, underlying processes;

2) Emergent properties are somehow autonomous from underlying processes (Bedau 1997: 376). 
From one side, the concept of emergence has been invoked to give higher-level properties a causal status, that is, placing them in the causal order. This is an ultimate consequence of Bedau's first point, the fact that emergent properties are constituted by underlying processes. These underlying processes must somehow interact both with other emergent and with non-emergent properties, thus sharing, at least part, their causal structure. It is in this latter respect that emergentism is different from epiphenomenalism. Indeed, having properties emerging and not playing any causal role is tantamount to proposing a form of epiphenomenalism, the view that some properties are effects but not causes of other properties. From the other side, the concept of emergence has been invoked to give some properties an autonomous status, to recognize them as properties of their own. This is the anti-reductionist thread, the second point in Bedau's quote, the view that emergent properties are somehow "more" or "different" with respect to the base properties from which they emerge.

It is the conjunction of these two features of emergent properties that is at the origin of many discussions and difficulties arising from this topic. Emergent properties are different from their base properties and they somehow take part in their causal order nevertheless.

In particular, the problem of having higher-level properties exercising causal influence on lowerlevel properties, the so-called downward causation, is one of the thorniest issue for those who want to defend the very idea of emergence. The appeal to downward causation is needed to avoid a host of difficulties. Suppose, in fact, that mental properties emerge from physical properties, are not reducible to these but are causally related solely and only to other mental properties. In what sense, then, is my raising my arm voluntary, when I claim it is, if my will is severed from or causally inefficacious with respect to, my physical movements? Similarly, how can we say that it is in virtue of what I have perceived that I have the belief I declare to have? In order to have mental properties playing a causal role, downward causation comes in handy, with causal influence cutting levels across.

The resulting picture is the following. Mental properties emerge from physical (neural) properties. They are not identical to physical properties, so these are autonomous from them, and do form a level of their own; at the same time, they can exercise causal influence on physical properties. So, there must be a form of downward causation, or causation from above. Given this general picture, I wish to raise a problem for it.

If the causal efficacy of emergent properties is defended, downward causation has to be accepted. Both emergence and downward causation presuppose the presence of levels of reality. However, emergence and downward causation pull in opposite directions with respect to my best reconstruction of what levels are. The upshot is that emergence stresses the autonomy among levels while downward causation puts the distinction between levels at risk of a reductio ad absurdum, with the further consequence of blurring the very notion of downward. Thus, emergence and downward causation are not a match for each other vis-à-vis the layered view of reality licensed by the concept of level. In order to argue for this, first I need to be clear on what emergence is and what the desiderata are for it.

\section{Desiderata for Emergence}

Established as a philosophical thesis in the mid of the XIX century (see McLaughlin 1992 and O'Connor and Wong 2015) emergentism posits the presence of properties that arise from the interaction of other properties but cannot be reduced to such properties and their interactions. The 
degree of autonomy that emergent properties have with respect to their base is part and parcel of the definition of emergent properties one provides. So, for instance, McLaughlin (1997: 39) says:

If $P$ is a property of $w$, then $P$ is emergent if and only if (1) $P$ supervenes with nomological necessity, but not with logical necessity, on properties the parts of $w$ have taken separately or in other combinations; and (2) some of the supervenience principles linking properties of the parts of $w$ with $w$ 's having $P$ are fundamental laws. ${ }^{1}$

This is known as supervenient emergentism, and aims at being a form of ontological emergentism, along the lines suggested by J. S. Mill and C. D. Broad, and pursued by van Cleve (1990), among others. According to this definition of emergentism, then, autonomy is somehow diminished because it seems somehow possible to trace back the causal effects of the emergent properties to the combined effect of their interacting parts. If this were the case, then there would be no place for any novel power to emerge, where novelty is a way to give a sense to autonomy (see Wong 2010).

A stronger version of emergentism takes emerging properties as "non-structural" properties, that is, properties that cannot be reduced to the properties and relations of their interacting elements. We may further analyze such a view by saying that the causal powers bestowed on an entity by the base properties and relations are superseded or transcended by the causal powers bestowed on that entity by the emerging property. Humphrey (1997a, 1997b) has advanced the idea that emergent properties are the result of some fusion process, one in which the original basal properties are lost and a new property occurs. This fusion process may be taken literally, as a physical process, for instance one that may occur at quantum level (O'Connor and Wong 2015; Wong 2006) or as due to the complexity of the system in which the properties at stake occur. Along similar tracks, O'Connor (2000) and O'Connor and Wong (2005) hold that emergent properties are the causal result of dynamical processes, thus abandoning the idea that emergent properties supervene on their synchronous basal properties and conditions. The autonomy of emergent properties is thus stressed by insisting on the idea that these properties grant new causal powers, powers that cannot be traced back to those of their base properties. Both views of emergent properties, the supervenient and the non-structural ones, share a number of desiderata for these properties, which could be useful to have clearly stated. These desiderata can be split in two groups. First, there are the desiderata for emergence properties (EPs) qua properties, and these are:

1) Emergent properties have a causal role;

2) The causal role of emergent properties is distinctive of them (it is different from that of their base properties);

3) The causal role of emergent properties is robust (laws involving).

Secondly, we have the desiderata for EPs qua emergent, and these are:

4) Emergent properties confer new causal powers;

5) The new causal powers of emergent properties are not deducible from the causal powers of the base properties and their relations.

We have considered properties in terms of causal roles or causal powers, as held by Shoemaker $(1980,2007)$. As we have seen, the gist of being an emergent property lies, in part, in bringing about new causal powers. However, this is ambiguous with respect to two different readings of the novelty of the powers with respect to the emergence of the property. There are two possible

${ }^{1}$ A law is fundamental if and only if it is not metaphysically necessitated by any other laws. 
readings: 1) An emergent property is one that confers causal powers that have never been instantiated in this nomological world, were it not for the base properties from which it emerges; 2) an emergent property is one that confers a new set of old causal powers, that is, that brings about causal powers that were already being instantiated in isolation, but never in conjunction. We may call these two readings, respectively, a-emergence and b-emergence. One may discard b-emergence as emergence inasmuch as it doesn't meet the desideratum of non-deducibility, a desideratum taken to be essential for something to be emergent. However, it may happen that something acquires, for whatever reason, a power that it didn't have, that was already present in its nomological world and such that the acquisition was not deducible. So, cases of b-emergence can be cases of emergence all the way down. Clearly, the desideratum of non-deducibility is trivially satisfied in case of aemergence. So, in what follow, I will consider emergence in the sense of a-emergence.

The crucial desiderata are \#3, \#4 and \#5. If the causal powers of the emergent properties are robust enough to involve laws or support counterfactuals, as in \#3, and these powers are new, \#4, it follows that these properties involve new laws, that cover the kind of causal relations determined by the new causal powers these properties confer to their bearers. This is something that was clear in emergentists such as Broad, who were speaking of "trans-ordinal" laws, as we will see. The relation between \# 3 and \#4, therefore, involves the idea that emergent properties come with their emergent laws. ${ }^{2}$ These laws regulate the relation between the base and the emergent properties. In particular, if one aims at having emergent properties that are non-structural, one cannot derive the causal powers of the emergent properties by simply considering the causal powers of their base properties and their relations: the law would take the relation to be a primitive one. A second and related feature is that meeting desideratum \#5 amounts to satisfying desideratum \#2. When it is claimed that emergent properties are non-structural, what is being stressed is that the new causal powers are such in virtue of not being deducible or predictable from the causal powers of their base properties, which make these powers distinctive of the new properties, as requested by desideratum \#2. Note here that the idea of construing the concept of emergence in terms of "novelty" and "unpredictability" presupposes a reference to our knowledge of their base level (Chalmers 1996, 2006; Kim 1999). Finally, desideratum \#1 avoids the risk of epiphenomenalism, while \#2 copes with the reductionist issue, which resurfaces also in \#5.

An interesting issue is whether meeting these desiderata guarantees that an emergent property is stable. For, you can have a property that is novel and unpredictable but is instantiated just once or randomly. What people have in mind in making sense of emergence (to borrow from Kim 1999) are properties which are novel, unpredictable and stable, that is, properties that occur whenever certain conditions are reached, which is what is pointed out by desideratum \#3. Those who favor the supervenient view of emergence have this further requirement satisfied by having the supervenience relation in place along with fundamental laws. For, if an emergent property is one that supervenes with nomological necessity on its base properties, then the stability of this property ensues. ${ }^{3}$ If, on the other hand, emergent properties are thought to be the result of some nonstructural relation, such as a fusion process or the like, then there is no guarantee that whenever the non-structural or fusion process occurs the emergent property ensues. If a property cannot be constantly associated with some condition, then it is not an emergent property. Hence, in this case too, some fundamental law is needed. Now that the main lines concerning the concept of emergence have been given, in order to elucidate downward causation, we should turn to the concept of causation.

\footnotetext{
2 In case of b-emergence, the conjunction of old laws can meet this desideratum.

${ }^{3}$ See on this, the debate between Kim (2006) and Marras (2006).
} 


\section{Construing causation}

Ned Hall (2004) has isolated two different conceptions of causation, a productive and a conceptual one. The distinction between these two conceptions of causation is given, as Hall has suggested, ${ }^{4}$ either in terms of transfer of energy, and this is the production conception, or in terms of some form of dependence, notably counterfactual dependence. ${ }^{5}$ Those who accept the idea of having emergent properties playing some causal role, endorse a counterfactual or conceptual view of causation. Those ready to reject the very idea of emergence, tend rather toward a productive or physicalistic view of causation. That this contrast is taken to be the one that determines many of the trouble of downward causation is witnessed by the recent exchange between Loewer and Kim (in McLaughlin and Cohen eds. 2007). On the one side, $\operatorname{Kim}(1998,2007)$ insists that dependence causation is not the final answer in that it can be accepted but still does not say in virtue of what it holds true. "Merely to point to the apparent truth, and acceptability of certain mind-body counterfactuals as a vindication of the mind-body causation is to misconstrue the philosophical task at hand" (Kim 1998: 43). The philosophical task to which Kim alludes to is to give an answer as to why these counterfactuals hold, that is to say, to find the relevant truthmakers. The importance of identifying the truthmakers of these counterfactuals is crucial in order to avoid epiphenomenalist worries, and "[f] undamentally these worries arise, I believe, from the question whether mentality has the power to bring about its effects in a continuous process of generation and production" (Kim 2007: 236). So, for Kim, the counterfactual conception stops midway, even if it is fine up to the point it reaches.

On the other side, Loewer (2007) insists that if causation is understood as production, Kim's so called "exclusion argument" - conceived to show the causal inefficacy of emergent or higher-level properties with respect to lower-level ones - can be successful, but at the cost of leaving also the brain inefficacious. The problem lies in how to single out the causing event. For, if what determines an effect is the event responsible for the transfer of energy, then such an event includes all the happenings belonging to the imaginary sphere determined by the speed of light one second before the event effect, considering the "one second before" as the minimum time interval needed to isolate the cause of an event. The radius of such a sphere is 186,000 miles and its center is the space-time region which we would call the cause of the event. "The upshot of this discussion is that if production is understood literally then perhaps Exclusion holds, but neither brain events nor mental events are producers of bodily movements" (Loewer 2007: 254). Endorsing the view of causation as dependence, on the contrary, blocks the Exclusion argument from the very onset while endorsing a view, counterfactual dependence, which is compatible with physicalism. ${ }^{6}$

Now, I agree with Kim that the dependence view still owes us an answer regarding why a certain counterfactual relation holds ${ }^{7}$; however, I have mentioned these two conceptions of causation because the plausibility of downward causation is often traced back to the acceptance of the dependence view of causation and, at the same time, the difficulties of downward causation are identified with the acceptance of the productive view. So, one could think that, if a dependence view of causation is preferred, many of the problems related to downward causation will vanish.

\footnotetext{
${ }^{4}$ In this he is not alone: see also Shaffer (2003) and Dowe (2000) for similar distinctions.

${ }_{5}$ We may take Dowe and Kim as representative of the first view and Lewis and Kroedel of the second one.

6 The best attempts to defend compatibilism against the overdetermination threat, which follows from the exclusion argument, are in Bennett $(2003,2008)$

${ }^{7}$ See Crane (2001) for a different but nevertheless skeptical perspective on counterfactual theories of causation.
} 
On the contrary, I think that emergence and downward causation are not well suited to each other even if we accept a dependence or counterfactual view of causation.

Let's grant, then, that, if the new powers of some emergent property do some causal work, this is to be traced to their sustaining some counterfactual of the form: Had [emergent property EP here] not occurred, [some property $\mathrm{P}$ here] would not have occurred either. In such a case, $\mathrm{P}$ counterfactually depends on the emergent property EP. Moreover, the effect of the causal relation, $\mathrm{P}$, may well be an emergent property as well. So, let's admit, with the defender of downward causation, that counterfactual causation is causation enough.

\section{What are levels?}

Both the concept of emergence and the concept of downward causation implies the presence of levels. However, levels are strained in opposite directions by the two concepts. Emergence, on the one side, pulls in the direction of making properties at one level autonomous with respect to properties belonging to a different level; downward causation, on the other side, points out that the causal efficacy of properties cuts across and holds between levels, so that levels are the tie for the efficacy of emergent properties. Basically, we are following the issue as set up by Bedau, this time metaphysically, for we are facing the fact that the level talk crosscuts the realization talk.

How can we capture the elusive concept of level? In recent times John Heil (2003) has strongly criticized this concept, arguing that it is the metaphysical consequence of a wrong semantic view. But abandoning the very concept of level would determine the collapse of the whole emergence project. So, I will try to give a sense to it. Broad, in defining "trans-ordinal" law, as opposed to the "intra-ordinal" one, sketched what levels are ("orders" in his terms) by arguing for a compositionalmereological view:

A trans-ordinal law would be one which connects the properties of aggregates of adjacent orders. A and B would be adjacent, and in ascending order, if every aggregate of order B is composed of aggregates of order $\mathrm{A}$, and if it has certain properties which no aggregate of order A possesses and which cannot be deduced from the A-properties and the structure of the B-complex by any law of composition which has manifested itself at lower-levels. (Broad 1925: 77).

So, the thesis is: if an aggregate has a property that is not present in its aggregate composing elements, then the two aggregates are adjacent and in ascending order. It seems to me that this view doesn't fare well with the fusion process view that is heralded and defended by Humphrey and by those who argue in favor of strong ontological emergence. For, the concept of aggregation presupposes the possibility of recognizing parts and relations, which is what is excluded by the fusion process, a process whose final result doesn't allow you to disentangle the contribution of the original parts and relations from the causal power of the new entity. A second reason for excluding the compositional view as the one to which the concept of level should be analysed, is the following. We know that some aggregate is adjacent to a given lower level if that aggregate has a different property from the aggregates of its part. However, it is possible that an aggregate can be multiply realized, that is, it is possible that the higher-level aggregate could be composed of aggregates of different types, none of which has the properties the higher-level aggregate has and such that these aggregates are different from each other and, possibly, in an aggregation relation as well. Imagine three levels, C (highest), B and A (lowest). C could be multiply realized by B and Aaggregates none of which has property $\mathrm{P}$, manifested by $\mathrm{C}$-aggregates. For instance, imagine that C-aggregates (groups of mammals) have properties like collaborative collective purposes. It is 
possible that B-aggregates have psychological properties such to determine collaborative collective purposes at level C. It is also possible, though, that without having psychological properties at all, biological individuals got viruses in their cells, such to determine collaborative collective purposes. The scenario I am imaging is the one presented in $W$ orld $W$ ar $Z$ (a novel written by Max Brooks in 2006, then a movie directed by Marc Forster in 2013) where people get a virus that transforms them in zombies, making their social behavior autonomous from their psychological properties, but similar to that of purposeful beings. Therefore, properties manifested by $\mathrm{C}$-aggregates can be realized by $\mathrm{B}$ and A-aggregates. Moreover, A-aggregates (not having neither property $\mathrm{P}$ nor $\mathrm{Q}$ ) compose both B-aggregates (having property $\mathrm{Q}$ ) and C-aggregates (having property $\mathrm{P}$ ), and Baggregates compose C-aggregates. Now, both A and B-aggregates lack the very same property which is present once $\mathrm{C}$-aggregates are in order. Which level is adjacent to $\mathrm{C}$ provided that $\mathrm{B}$ aggregates are different form A-aggregates? We do not have a clear way to answer. Beside, we should rule out that both $\mathrm{A}$ and $\mathrm{B}$ are adjacent, otherwise adjacency would be transitive, which is not the case. ${ }^{8}$

In order to understand what levels are, I propose to use the concept of causal law as the pivotal concept on which levels are individuated. The intuitive idea is that two properties $\mathrm{P}$ and $\mathrm{P} *$ belong to one and the same level if and only if there is a causal relation connecting $\mathrm{P}$ and $\mathrm{P*}$. Physical properties are involved in causal relations with other physical properties and the same holds for chemical or biological properties. So, each group of properties that forms a sort of holistic network of potential or actual causal interactions belongs to one and the same level. However, this interpretation of levels would immediately create a tension for emergent properties vis-à-vis their supposed downward effects. For, if these properties establish causal relations with properties at a lower-level, that would be a reductio ad absurdum of the concept of level thus analyzed. For instance, if a biological property has effects on a physical property, given the proposed analysis, since causal interaction among properties guarantees membership to the same level, the biological level and the physical level would collapse into one, and we should deny that the biological property is biological, in virtue of the effect it can have on a physical property (or the other way around), thus eliminating from the very beginning the possibility of downward causation. It seems that this proposal is not viable. A better proposal follows these guiding principles: presumably, properties belonging to different levels form disjunctive sets. We need a way to assign each property only to one level. Since downward causation is a tie between levels, such tie must be different from the ties that occur intra-level. Moreover, it has been assumed that the laws that connect properties belonging to different levels are new fundamental laws, as it has been stressed by O'Connor and Wang (2005) ${ }^{9}$ and then elaborated by Wong (2010). At the same time, we know that there are many fundamental laws that occur within single levels, as is the case with physics. How can we tell laws that are fundamental and intra-level from those that are inter-level? Let's assume that fundamental laws

${ }^{8}$ Carl Gillett has new and interesting views on realization and composition, which I will not consider here. See Gillett (2003).

${ }_{9}^{9}$ O'Connor and Wong (2005) insist that emergent properties do not supervene on their base properties because emergent property are caused diachronically by those base properties that satisfy certain complexity requirements. And emergent properties do not supervene on physical properties neither strongly nor globally, given the possibility of probabilistic causal connections. However, in adopting this line of thought is not clear what is the relation between the emerging property and the base which happens to be synchronous with the emergent property, which is different from its causing base (which is asynchronous with respect to the emerging property, since it causes it). For, if an emergent property can cause a change in a lower-level property, we are back to the reductio imagined. Moreover, we seem to be back to the problem raised by Kim's exclusion argument, because we may ask what causal job is done by the base properties that occur moments before the change in their own causal level. If they do not do any work, then they would be epiphenomenal base properties; if they do a partial job, one that is not sufficient for the causal change to occur, we may have a case of pre-empted property; if, finally, they do all the causal job, then are the emergent properties that result epiphenomenal? 
occur between levels, while non-fundamental laws occur within levels. Non-fundamental laws are necessitated by other laws while fundamental laws are not necessitated by any other law. Therefore, fundamental laws are the counterparts of what Broad called trans-ordinal laws, even though in his case lower-level properties constituted the antecedent of the law, while emergent and higher-level properties were the consequent of the law. Let's set a definition for levels:

Levels: Two properties P and P* belong to one and the same level if and only if (i) they are both covered by one non-fundamental causal law; (ii) they are indirectly covered by other nonfundamental causal laws; (iii) the causal relation is non-transitive.

Let me elucidate the above definition. Consider properties $\mathrm{P}$ and $\mathrm{P} *$ : they belong to the same level if and only if either there is a non-fundamental law such that $\mathrm{P}=>\mathrm{P}^{*} \cdot{ }^{10}$ Regarding the second clause: $\mathrm{P}$ and $\mathrm{P}^{*}$ belong to the same level if there are laws such that $\mathrm{P}=>\mathrm{Q}, \mathrm{P} *=\mathrm{Q}^{*}$ and $\mathrm{Q}=>\mathrm{Q}^{*}$, thus satisfying indirectly the fact that $\mathrm{P}$ and $\mathrm{P} *$ belong to the same level. In the definition, I have mentioned non-fundamental law. Fundamental laws are those that are not necessitated by other laws but necessitate non-fundamental laws. So, if $\mathrm{P}$ and $\mathrm{P} *$ are covered by a fundamental law, they belong to the same level only if they are covered by further non-fundamental laws. For instance, if $\mathrm{P}=>\mathrm{P}^{*}$ is fundamental, then $\mathrm{P}$ and $\mathrm{P}^{*}$ belong to the same level if there are non-fundamental laws that cover $\mathrm{P}$ and $\mathrm{P}^{*}$ and are such to connect them indirectly -- that is, in virtue of other laws. We may say that there must be an epistemological path connecting laws that cover both $\mathrm{P}$ and $\mathrm{P} *$. In such a case, the law is fundamental for the level at stake. Vice versa, if two properties are connected solely by a fundamental law, and are mentioned in two different sets of laws, then these do not belong to the same level; consequently, the fundamental law is an inter-level one. Finally, the third clause should be self-evident, but I will say something shortly.

The definition provided is based on the presence of some law, rather than on a simple causal interaction, to determine whether two properties belong to the same level. So, the definition allows for the possibility of cases of singular causation that cuts across levels without touching the robustness of the concept of level, if these cases are not backed up by some law. It is thus possible that specific cases of downward causation occur without undermining the distinction of levels. Besides, given the possibility of a certain amount of independence between properties intuitively belonging to the same level (for instance, mechanical and thermo-dynamical physical properties), the definition allows that other properties (e.g. statistical-mechanical) may play intermediate roles to grant membership to the same level. For instance, properties $\mathrm{P}, \mathrm{P}^{*}$ and $\mathrm{P} * *$ belong to the same level even if there is no specific law that connects $\mathrm{P}$ and $\mathrm{P}^{* *}$, provided that there are at least laws that connect $\mathrm{P}$ to $\mathrm{P}^{*}$ and $\mathrm{P} *$ to $\mathrm{P}^{* *}$ respectively. In this way, all the properties belonging to the same level form a sort of interrelated holistic network of laws, one that makes the concept of level, and the metaphysics behind it, robust enough.

Finally, since laws can be interpreted as groups of counterfactuals, and since many think that counterfactuals are not transitive (Hitchcock 2001), then we cannot assume transitive relations among properties mentioned in connected laws unless these are mentioned in some law. The definition then, describe the presence of an interconnected network of laws, without determining that between the elements of such a network transitivity must hold. So, one can imagine that level $n$ comprises laws $\mathrm{L}_{1}, \mathrm{~L}_{2}, \ldots \mathrm{L}_{\mathrm{n}}$ such that some properties mentioned in law $\mathrm{L}_{\mathrm{i}}$ are also mentioned in law $\mathrm{L}_{\mathrm{j}}$ without this entailing that the different properties mentioned in laws $\mathrm{L}_{\mathrm{i}}$ and in law $\mathrm{L}_{\mathrm{j}}$ can be

${ }^{10}$ Read the sign "=>" as the "cause" relation. 
subsumed under a new law in virtue of transitivity relations. A specific law covering the new relation is required, deductions cannot do the work. However, transitivity holds between laws. In sum, I'm granting this analysis and definition to my opponent.

Let us see how the definition fares with the problems of emergence and downward causation. Consider psychophysical laws: these establish counterfactual dependencies between physical and psychological properties, dependencies that are taken to be sufficient by those who defend downward causation (see Kroedel 2015a; 2015b). For instance, so-called Fechner's law establishes that subjective sensation is proportional to the logarithm of the stimulus intensity. ${ }^{11}$ It determines counterfactuals such as: had the stimulus intensity being tripled the subjective sensation would have doubled. Now, the relevant law can be expressed as: physical stimuli of type P cause this level of sensation $\mathrm{S}, \mathrm{P}=>\mathrm{S}$. In order to cut across levels, this law must be of fundamental kind. This law necessitates other laws that either mention $\mathrm{P}$, or mention $\mathrm{S}$, but do not mention $\mathrm{P}$ and $\mathrm{S}$ together, if $\mathrm{P}$ and $\mathrm{S}$ are to belong to different levels. So, the properties that are nomologically connected to the physical property $\mathrm{P}$ and those that are connected to the psychological property $\mathrm{S}$ form two disjoint sets, to which the difference in levels amounts.

The general picture that we gained is a Humean one: all properties are nomologically connected to other properties in a very local way, in virtue of being mentioned in laws. The properties that are covered by many laws form a network and this network is what determines level membership. It is possible to connect levels by having fundamental laws covering properties that are connected with different networks, that is, that belong to different levels; the reductio of the concept of level is prevented. Not having transitivity is crucial for having emergence, because it blocks possible deductions of the powers of properties covered by more than one law.

The first problem lurking behind this picture, however, is the following: on the one side, all these laws are contingently related to one another; on the other side, we ask for an anti-necessitation relation between the properties mentioned in the fundamental laws. What we have, in fact, is that one property belonging to level $n$ cannot in isolation necessitate other properties belonging to level different from $n$ even if adjacent to it, on pain of blurring the distinction between levels and, in perspective, to bring to the envisaged reductio. For, if property $\mathrm{S}$, in the example given above, is covered by other laws that cover $\mathrm{P}$ or properties belonging to the same level as $\mathrm{P}$, laws different from $\mathrm{P}=>\mathrm{S}$, then how can we be prevented from saying that $\mathrm{S}$ belongs to the same level as $\mathrm{P}$, thus taking the fundamental law $\mathrm{P}=>\mathrm{S}$ as one belonging to the level to which $\mathrm{P}$ belongs? Clearly, the same should hold for including $\mathrm{P}$ in the level to which $\mathrm{S}$ belongs. This is the case not only for symmetrical reason, but also on pain of having $\mathrm{P}=>\mathrm{S}$ belonging to two different levels, those of $\mathrm{P}$ and S. Which would be just few steps before saying that the level is just one, precipitating again into the reductio. On the other hand, if $\mathrm{P}$ and $\mathrm{S}$ are covered by other laws that cover subsets of the properties belonging to the levels to which $\mathrm{P}$ and $\mathrm{S}$ belong, they may form a new level placed in between that of $\mathrm{P}$ and that of $\mathrm{S}$, determining a new level between that to which $\mathrm{P}$ and $\mathrm{S}$ belongs, respectively. However, it would be possible to have this process repeated again and again, thus generating a continuum of levels. Such a continuity, though, would be a problem for having properties emergence: we would end up by having levels emergence, but for it we would need a different analysis on the concept of emergence. Finally, meeting the condition of having levels clearly differentiated constitutes a non-contingent requirement on the modal structure of levels, one which runs against the general Humean picture we have imagined in the attempt to be charitable toward the defender of downward causation and emergence.

${ }^{11}$ Fechner, or Weber-Fechner law, has now been superseded by Stevens' power law. 


\section{A case of emergence?}

Let me offer an example in order to better clarify the issue. Consider new human-machine interfaces. Thanks to special detectors, like a cap placed on the skull, the electrical activity of the brain is recorded and converted into computer signals. By means of these signals, the subject can interact with some other electric devices, giving commands and moving it. In order to accomplish such results, the subject has to train her or himself to think so-and-so in order to express the command. Basically, one trains one's own mind to have one's brain working so-and-so, or this is the way an emergentist would put the issue.

Authors working in this field say: "Electrical signals coming from different parts of the human body can be used as command signals for controlling mechanical systems. However, it is necessary that the individual in charge of controlling such devices be able to intentionally generate such signals. It is also necessary that the interface adopted (the Human-Machine Interface - HMI) can 'understand' and process such signals, setting the command that better fits the wish of the individual." (Ferreira et al. 2008).

In such a case, what we have is, presumably, a case of ontological emergence, as envisaged by Humphrey and others, because the subject, at least prima facie, has acquired a new causal power that of acting on a digital device by intentionally directing his thoughts. We can grant to the emergentist the following depiction of the situation: first we have the development of a new emerging property (EP) of the mind from a property of the brain $(\mathrm{P})$ establishing a causal law such as: $\mathrm{P}=>\mathrm{EP}$. In this case, there would be a causal emergent relation. Then we have the action, where the new emerging state of the mind causally controls some machine through a computer, which is evidence of downward causation, because it brings about a physical property (P'). So, we have a three stages process, $\mathrm{P}=>\mathrm{EP}=>\mathrm{P}$ ', that is, a case of emergence and then one of downward causation, which is what is desired.

The problem I mentioned is as follow: defenders of emergence want emerging properties to have new causal powers (desideratum \#4) that cannot be deduced a priori (\#5), and so do not place specific restrictions on what could happen. Many laws may flourish, and the one mentioned in the example is a case in point. These new laws would cover physical and psychological properties, thus fixing the causal relations between these two levels. However, with the flourishing of these laws, what remains of the concept of level? Given the metaphysical picture determined by the proposed analysis of levels, this activity of the mind cannot become general and proliferate, for it would determine the collapse of the distinction between levels. For having many types of conscious properties determining many different types of causal effects on physical properties would determine many new fundamental laws connecting the two levels, in a number so high that it would be impossible to distinguish the laws that set the levels from the laws that violate the levels distinction because new and fundamental. Basically, each emergent relation would be a primitive law, connecting the two levels. So, the more emergent properties arise and the more causally efficacious these are, in a stable and counterfactuals supporting way, the less it is possible to determine whether there are levels and so whether there is downward causation. This difficulty is tied to the presence of many different types of mental and physical properties, that can be linked via new emerging laws. This point brings us to a second problem.

What I have assumed so far is that there is a direct and unique link between a physical property and an emergent mental property. Basically, I've tacitly assumed that given an emergent property $\mathrm{E}$ there is only one base property from which it emerges. This base property can be a complex or 
a property plus a set of conditions, but this would not change the issue: we would have a one-toone relation, that could be also of causal sort, between a base and an emergent property. Now the issue is: what happens if emergent properties are multiply realizable? Let me expand this problem.

\section{Realization and emergence}

As we have seen, requirements \#2 and \#4 entail that emergent properties are distinct from their base properties. One option is the one I described before: a one-to-one relation between base and emergent properties, where these are different and irreducible to each other but related in a unique way, perhaps by strong supervenience or by fusion or what not. However, one may wonder whether these properties can also be multiply realizable. Indeed, one of the most important arguments against the type-identity between mental and physical properties was the multiple realizability one (Putnam 1960). Since many argue that mental properties are emergent properties, it is reasonable to consider whether these two concepts, emergence and multiple realizability, fare well with each other.

Consider how the dialectic since Fodor's famous paper "Special sciences" (1974) is usually set. The tokening of property $\mathrm{C}$ causes the tokening of property $\mathrm{E}$. Now, we should add that $\mathrm{C}$ and $\mathrm{E}$ belong to different levels, presumably $\mathrm{C}$ to a level higher than E's, so that downward causation occurs as well. Now, what Fodor and, I believe, Kim's schemas are telling us, is that causation occurs between token properties while causal explanation occurs between type properties. However, the various realizers of $\mathrm{C}$, like those of $\mathrm{E}$, should belong to the same level of their type, whichever level that happens to be. The reason for this is that, if it is the emerging property to be the cause, to be the one that plays the C-role in the causal explanation, thus satisfying desiderata \#1 and \#2, and the physical (non-emerging) property is to be the effect, playing the E-role, then the realizers of the emergent property have to be emergent tokens as well. For, if the realizers of the emerging property are not on the same ontological level of the type property they realize, the emergence in question would be only epistemological, not ontological. That is to say, if the realizing tokens of an emergent property were not on the same level of the emergent property, in particular if they were on the same level of the physical effect, they would count as realizers of the emergent property only in virtue of the epistemological advantage we would have in gathering or grouping them together under the same label.

To expand this point a little further: if the realizers of the emergent property weren't on the very same level of the property type, the causal relations would occur, presumably, at the same level of the effect property, and of its realizers, thus excluding the emergent property type from doing any causal work. This would have the consequence that there would be no need to postulate any downward causation at the ontological level. The "downwardness" of the causation would only be a façon de parler set for explanatory purposes, with no ontological significance, which, after all, is exactly the gist of Kim's exclusion argument. ${ }^{12}$ Let me elaborate.

Imagine an emergent property EP, say of mental sort, causing a lower-level property $\mathrm{P}$, of physical sort. The realizers of EP are the emergent tokens $\mathrm{ep}_{1}, \mathrm{ep}_{2}, \ldots$ e $\mathrm{p}_{\mathrm{n}}$, causing $\mathrm{P}$ to occur. Each realizer of EP may determine many potential downward causal relations $r_{1}, r_{2}, \ldots r_{n}$ depending on how the effect is brought about. For instance, if it is the case that $\mathrm{ep}_{1}=>\mathrm{P}$, call $\mathrm{r}_{1}$ the relation in question; since it could have been the case that $\mathrm{ep}_{2}=>\mathrm{P}$, because $\mathrm{ep}_{2}$ counts as a realizer of EP as any other $\mathrm{ep}_{\mathrm{i}}$, then it could have been the case that $\mathrm{r}_{2}$, which is another of the many possible relations that

${ }^{12} \mathrm{It}$ is important to consider, however, the different moral that is drawn from this kind of problem by emergentists such as MacDonald and MacDonald (2010). 
the emergent property type EP could have realized in causing property type P. So, let's gather together all the possible relations as realizers of the relation type $\mathrm{R}$, that says that if a property of type EP occurs it causes a property of type $\mathrm{P}$ to occur as well, which is a robust law that satisfy desideratum \#3. So, no matter their differences, all the realizers of the property types have to comply with the counterfactuals generated by the law. Now, it is reasonable to consider that the imagined law, $E P=>P$, since it subsumes emerging property tokens that may be quite different one from the other, would be a very loose one, one that should be formulated in such a way as to cancel differences and specificities. Possibly, counterfactual dependences could still hold but Kim's point on the causal depth of counterfactuals resurfaces with new strength: how can we claim ontological emergence if this is guaranteed only by ontologically quite different counterfactuals whose only commonality is that of being subsumed under a general and sloppy law? As one can appreciate, the point rests on the multiple realizability assumption, the feature, so far, needed to block the idea that mental properties are identical with physical properties. Such an assumption is not necessary for the emergentist, she or he can claim the one to one correspondence imagined above, but it is a likely appeal if one consider the role this argument had and still has in the philosophy of mind.

\section{Conclusions}

The two opposite pulling threads mentioned by Bedau, considered at the beginning of this paper, are striking back. These form a conundrum of epistemological and ontological issues, one that is difficult to assess given the nature of the problem: while emergence is ontological in character, and thus requires new properties and causal powers to arise, downward causation is epistemological, thereby leading to the expectation that new laws and counterfactuals can be truly stated. Both rely on the concept of level and this concept cannot be squared with the multiple realizability of emergent properties, as mental properties are taken to be. I have tried to explore the metaphysical side of this conundrum, but these two threads remain entangled and pulling in opposite directions. Emergence and downward causation do not fit each other.

\section{Acknoledgements}

I wish to thank Francesco Orilia and Michele Paolini Paoletti for a thoughtful reading of a previous version of this paper.

\section{References}

Bedau, M. (1997) "Weak Emergence”, Philosophical Perspectives 11: 375-99.

Bennett, K. (2003) "Why the exclusion problem seems intractable, and how, just maybe, to tract it", Noûs, 37: 471-97.

Bennett, K. (2008) "Exclusion again", in J. Hohwy and J. Kallestrup (eds) Being Reduced, Oxford University Press.

Broad, C. D. (1925). The Mind and Its Place in Nature. London: Routledge and Kegan Paul.

Chalmers, D. (1996) The Conscious Mind: In Search of a Theory of Conscious Experience, New York: Oxford University Press.

Chalmers, D. (2006) "Strong and Weak Emergence," in Clayton and Davies (2006)

Crane, T. (2001). The significance of emergence. In C. Gillett and B. Loewer (eds), Physicalism and Its Discontents (pp. 207-224). Cambridge: Cambridge University Press,.

Dowe P. (2000) Physical Causation, Cambridge University Press, Oxford

Ferreira et al. (2008) "Human-machine interfaces based on EMG and EEG applied to robotic systems" Journal of NeuroEngineering and Rehabilitation, 5:10 DOI: 10.1186/1743-0003-5-10. 
Fodor, J. (1974) “Special Sciences," Synthese, 28: 97-115.

Gillett, C. (2003) "The Metaphysics of Realization, Multiple Realizability, and the Special Sciences", The Journal of Philosophy, 100: 591-603.

Hall, N. (2004) "Two concepts of causation" in J. Collins, N. Hall, L.A. Paul (eds) Causation and Counterfactuals, Cambridge Ma: Mit Press.

Heil, J. (2003) From an Ontological Point of View, Oxford University Press, Oxford.

Hitchcock, C. (2001) “The intransitivity of causation revealed in equations and graphs", Journal of Philosophy 98: 273-99.

Humphrey (1997a). "How Properties Emerge," Philosophy of Science, 64: 1-17.

Humphrey (1997b). "Emergence, Not Supervenience," Philosophy of Science, 64: S337-S345.

Kim, J. (1998) Mind in a physical world, MIT Press, Cambridge MA.

Kim, J. (1999) "Making Sense of Emergence" Philosophical Studies, 95: 3-36.

Kim, J. (2006) "Emergence: Core Ideas and Issues", Synthese 151: 547-59.

Kim, J. (2007) "Causation and mental causation" in B. McLaughlin and J. Cohen (eds) Contemporary debates in Philosophy of mind, Oxford: Blackwell: 227-42.

Kroedel, T. (2015a) "A simple argument for downward causation", Synthese 192: 841-58.

Kroedel, T. (2015b) "Dualist mental causation and the exclusion problem", Nồs 49 (2): 357-75.

Loewer, B. (2007) "Mental causation, or something near enough" in B. McLaughlin and J. Cohen (eds) Contemporary debates in Philosophy of Mind, Blackwell, Oxford: 243-64.

MacDonald C. and MacDonald G. (2010) "Emergence and Downward Causation" in MacDonald, C. and G. MacDonald, eds., Emergence in Mind, Oxford University Press, New York: 139-68.

McLaughlin, B. (1992) "The Rise and Fall of British Emergentism," in A. Beckermann, H. Flohr and J. Kim (eds) Emergence or Reduction? Essays on the Prospect of Non-Reductive Physicalism, De Gruyter, Berlin: 49-93.

McLaughlin, B. (1997) “Emergence and Supervenience," Intellectica, 2: 25-43.

Marras, A. (2006) "Emergence and Reductionism: reply to Kim", Synthese 151: 561-69.

O'Connor, T. (2000) “Causality, Mind and Free Will,” Philosophical Perspectives, 14: 105-117.

O'Connor, T. and Wong, H. Y. (2005) “The Metaphysics of Emergence”, Noûs, 39: 658-678.

O'Connor, T. and Wong, H. Y. (2015) "Emergent Properties", The Stanford Encyclopedia of Philosophy

(Summer 2015 Edition), Edward N. Zalta (ed.), URL

http://plato.stanford.edu/archives/sum2015/entries/properties-emergent/.

Putnam, H. "Minds and Machines", now in H. Putnam (1975) Mind, Language and Reality, Cambridge University Press, Cambridge MA.

Schaffer J. (2003) "The Metaphysics of Causation", The Stanford Encyclopedia of Pbilosophy, (Winter 2003 edition), Edward N. Zalta (ed.), URL http://plato.stanford.edu/entries/causationmetaphysics/

Shoemaker, S. (1980) "Causality and properties", reprinted in J. Kim and E. Sosa 1999, (Eds.), Metaphysics. An Anthology, Blackwell, Oxford, pp. 253-68.

Shoemaker, S. (2007) Physical Realization, Oxford University Press, Oxford.

Van Cleve, J. (1990) "Mind-Dust or Magic? Panpsychism Versus Emergence" in Philosophical Perspectives (4), J.

Tomberlin (ed.), Ridgeview, Atascadero CA: 215-26.

Wong, H. Y. (2006) "Emergents from Fusion," Philosophy of Science, 73: 345-367.

Wong, H. Y. (2010) “The Secret Lives of Emergents," in A. Corradini and T. O'Connor (eds.)

Emergence in Science and Philosophy, Routledge, New York: 7-24. 